\title{
Classification of various types of disability and determining their predictive causes in western Iran
}

\author{
Ali Zakiei ${ }^{\mathrm{a}}$, Narges Kiani ${ }^{\mathrm{b}}$, Faramarz Morovati ${ }^{\mathrm{c}}$, Saeid Komasi ${ }^{\mathrm{d} \text {,* }}$ \\ ${ }^{a}$ Sleep Disorders Research Center, Kermanshah University of Medical Sciences, Kermanshah, Iran \\ ${ }^{\mathrm{b}}$ Department of Welfare of Kermanshah Province, Kermanshah, Iran \\ ${ }^{\mathrm{c}}$ Department of Counseling, Razi University, Kermanshah, Iran \\ ${ }^{\mathrm{d}}$ Clinical Research Development Center, Imam Reza Hospital, Kermanshah University of Medical Sciences, Kermanshah, Iran
}

A R T I C L E IN F O

\section{Keywords:}

Causations

Clustering

Disability

Epidemiology

\begin{abstract}
A B S T R A C T
Objectives: The current study aims to clustering different types of disability in Kermanshah Province and determine its predictive causes.

Methods: The statistical population of this cross-sectional study includes all the households living in rural and urban areas of Kermanshah Province in western Iran. Using multi-stage cluster sampling, 10150 households were selected from July to December of 2017 based on the population share of each city district. The required data were obtained using a 13-item questionnaire developed by the research team. The obtained data were analyzed using two-step cluster analysis, chi-square test, and multinomial logistic regression analysis.

Results: This model suggested five clusters including (i) geographically scattered subjects with mental abnormalities, (ii) geographically scattered subjects with physical motor abnormalities, (iii) non-eastern subjects with multiple abnormalities, (iv) non-northern subjects with multiple abnormalities, and (v) geographically scattered subjects with visual-auditory abnormalities. Multinomial logistic regression emphasized the significance of some causes of disability, particularly head trauma and trauma caused by car accidents $(\mathrm{P}<0.05)$. There was a significant difference among the clusters with regard to socio-demographic variables $(\mathrm{P}<0.05)$. Conclusion: The eastern areas of Kermanshah province are facing a larger number of mental disorders caused by mother's disease during pregnancy and cousin marriages. Other major causes of disability include head trauma and trauma caused by car accidents. In western areas, physical-motor disability is more frequent, and in central areas, visual-auditory abnormalities are more prevalent.
\end{abstract}

\section{Introduction}

The concept of disability has been defined in the international classification of functional disability and health by World Health Organization (WHO) as an umbrella term for functional and participatory injuries and limitations. ${ }^{1}$ Based on the definition of the World Health Organization, disability involves the lack of ability to perform ordinary and usual tasks, which is caused by impairment in a body organ. ${ }^{2}$ The majority of people with disability are marginalized in all aspects of their lives, they are more likely to be unemployed due to discrimination, and they usually have a very low quality of life. ${ }^{3}$ Despite the fact that the prevalence of four main disability including physical-motor, visual, auditory, and mental is reported to be $3.4 \%$ of the global population, ${ }^{4}$ the WHO has announced that about 1 billion people in the world (about 15\%) live with some form of disability. ${ }^{5}$
Moreover, the retrospective analysis of the data from the World Health Survey of 54 countries shows a $14 \%$ global prevalence for disability. ${ }^{6}$

Besides the fact that the prevalence of various disabilities around the globe does not follow an identical pattern, it seems that its causes are also completely dependent on the socioeconomic and geographical conditions of each country. ${ }^{7}$ Due to the wide range of disabilities in different ages; the causes of disability cannot be included in a single category. For example, the cause of disability in the elderly is more related to biological factors and other factors such as obesity and diabetes $^{8,9}$; While childhood disability may be related to infectious causes. $^{10}$

So far, there has been no comprehensive classification of various types of disability. Classification of various mental and physical disability will facilitate the identification of target groups in order to provide timely interventions and it will also help health policy makers

\footnotetext{
${ }^{*}$ Corresponding author. Clinical Research Development Center, Imam Reza Hospital, Kermanshah University of Medical Sciences, Zakarya Razi Boulevard, Kermanshah, Iran.

E-mail address: s_komasi63@yahoo.com (S. Komasi).
} 
to provide services based on the preferences and needs of the population. ${ }^{11,12}$ Cluster analysis is a highly useful statistical method for partitioning the population based on common characteristics and a wide range of mental and physical disability and disabilities. ${ }^{13,14}$ Since the phenomenon of disability and its negative physical, mental, and social consequences are considered as one of the main barriers facing the growth and development of countries, ${ }^{4}$ the classification of various types of disability in a country and evaluating their causes can be effective in the financial planning and health policy making of the country. Based on these considerations, the current study is carried out in order to clustering various types of disability in Kermanshah Province and to determine their predictive causes.

\section{Materials and methods}

\subsection{Design and setting}

The statistical population of the current cross-sectional study includes all the households living in rural and urban areas of Kermanshah Province with at least five years of residence in this province. Kermanshah Province is located in western Iran and based on the National Census of 2016, its population is 1,952,434 individuals, among which 988.015 are men and 964,419 are women. In general, $2.44 \%$ of the total population of the country lives in Kermanshah Province. According to the National Statistical Organization of Iran, there are 576,861 households in Kermanshah Province, among which $76 \%$ live in urban areas and $24 \%$ live in rural areas. The population statistics of each one of the cities in this province are presented in Table 1.

\subsection{Participants and sampling}

In order to carry out this study, 10,150 households from urban and rural areas of Kermanshah Province across all ages and genders were selected using multi-stage clustering. The criteria for clustering statistical blocks were based on the latest official census carried out by the National Statistical Organization of Iran. In each city, the samples were selected in proportion to the total population. Considering the large size of the statistical population and in order to minimize bias, Cohen's sample size formula at the $99 \%$ confidence level and 0.01 error rate was utilized. Finally, 10,150 households were entered into the study. The participation rate was $100 \%$.

\subsection{Gathering data}

In this survey study, in order to perform the interviews and gather the data, at first, 21 undergraduate and graduate students of psychology

Table 1

Ratio distribution of selected households based on population.

\begin{tabular}{lll}
\hline Name of the city & Number of selected households & Population percentage \\
\hline Islam-Abad Gharb & 700 & 7 \\
Gilan Gharb & 300 & 3 \\
Dalahou & 200 & 2 \\
Sare-Pole-Zahab & 400 & 4 \\
Ghasre Shirin & 150 & 1.5 \\
Harsin & 400 & 4 \\
Sahneh & 400 & 4 \\
Kangavar & 400 & 4 \\
Songhor & 450 & 4.5 \\
Kermanshah & 5600 & $\mathbf{5 6}$ \\
Salas Babajani & 200 & 2 \\
Javanroud & 400 & 4 \\
Paveh & 300 & $\mathbf{3}$ \\
Ravansar & 250 & 2 \\
Total & 10,150 & 100 \\
\hline
\end{tabular}

and rehabilitation consultation were trained by the research team in training courses as interviewers. These individuals went through five 1$\mathrm{h}$ training sessions to become familiar with various disabilities. The training was provided using books, slides, and videos by two psychotherapists. After training the interviewers, based on the familiarity with a specific region of the province, each one of them became responsible for gathering data at that region. Before starting the study, the household heads were asked for a written consent form for participating in the study and they were provided with the necessary assurance on the confidentiality of their information. The household heads or one of the parents was prioritized for answering the questionnaire. If necessary, other family members and the person or persons with disability were also interviewed. If the household had a medical file or document, those were also examined and evaluated. While this study utilized interviews and observations, the main data gathering tool was a 13-item questionnaire developed by the research team. The first section of the questionnaire included the demographic information of people with disability including age, gender, marital status, education level, and location, while the second section was developed based on the opinions of experts and previous studies to measure the disability and its related factors. In order to construct the questionnaire, after gathering a number of items based on available studies, the items were presented to three graduate psychology students, two psychology PhD's, a social welfare graduate, a health training graduate, and a rehabilitation physician. Then, the irrelevant items were eliminated and the final questionnaire was developed based on 13 items.

\subsection{Data analysis}

Before performing the main analysis, at first, the classification variables including the presence or lack of each one of disability was coded as no $(=0)$ /yes $(=1)$. The numbers of disability were also recorded and the living location was classified and recorded as the center, north, east, and south of the province. Considering the simultaneous presence of continuous and categorical variables, two-step cluster analysis (TSCA) was performed in order to identify the clusters. This method of analysis was used due to the large size of the sample and the presence of qualitative categorical variables. ${ }^{15}$ The TSCA determines the importance ranking of classification variables which affect the prediction of the model and it also automatically determines the number of clusters. The fitting of the model was measured using Schwarz's Bayesian Information Criterion (BIC) using the average silhouette coefficient. The silhouette coefficient is a measure of internal validity which ranges from 0 to 1 . Scores closer to 1 indicate the superiority of the model. ${ }^{15}$ Then, the dispersion of various types of disability among the clusters was calculated using the chi-square test.

As the next step, the multinomial logistic regression was used in order to identify the correlates of the derived clusters. The causes of disability (family marriage, disease during pregnancy, pregnancy malnutrition, mother's aging, family history of disability, illness during childhood, head-traffic-war trauma, and war side trauma), sex, and number of family members were entered into the model simultaneously. Considering the presence of five subgroups, the first cluster (having only mental disability) was considered as the reference cluster. The results of the analysis were presented as adjusted odds ratios (OR) with $95 \%$ confidence interval. As the final step, socio-demographic factors were identified in the population and based on the clusters and the statistical significance of the difference between the clusters with regards to these variables was calculated using the chi-square test. Moreover, in an extra level of analysis using single-variable chi-square test, each one of the clusters was compared to the reference cluster separately. In order to perform this comparison, the frequency of each cluster compared to the reference cluster was calculated and after determining the weights of the frequencies, the significance of the difference between the two clusters was calculated. All the statistical analyses were carried out using SPSS20 (IBM Corp., Armonk, NY, USA). 
All the tests had two-tailed and statistical significance was defined as $P$ value $<0.05$. The data related to continuous variables were presented as mean and standard deviation and categorical data were presented as values and percentages.

\section{Results}

\subsection{Identified clusters}

Table 2 presents the profile obtained from the two-step cluster analysis as well as the summary of the model. As can be seen from the table, the silhouette measure of cohesion and separation is completely acceptable. While the disability play the main role in determining the clusters, there is a significant difference between the clusters in terms of all three variables under study $(\mathrm{P}<0.0005)$. This model suggests five clusters as follows: (i) geographically scattered subjects with mental abnormalities, (ii) geographically scattered subjects with physical motor abnormalities, (iii) non-eastern subjects with multiple abnormalities, (iv) non-northern subjects with multiple abnormalities, and (v) geographically scattered subjects with visual-auditory abnormalities.

\subsection{Characteristics of the participants based on clusters and clusters' correlates}

Table 3 presents the information for each one of the clusters and the results of the multinomial logistic regression. The information in this table indicates that gender and causes of disability are significantly effective in predicting the clusters $(\mathrm{P}<0.05)$. Moreover, the summary of the model shows that this model has a good fit $(\mathrm{P}<0.0005)$ and it can predict $11.7-31.5 \%$ of the variance in the clusters (Table 3 ).

\subsubsection{Cluster 2 vs. cluster 1}

Compared to the reference cluster (people suffering from mental disorders), members of cluster 2 are more exposed to childhood disease, head trauma, and trauma caused by car accidents. Indeed, childhood disease and head trauma increase the likelihood of physical-motor disorders up to 3.4 to 17.5 times and head trauma increases this likelihood up to 97 times $(\mathrm{P}<0.05)$.

\subsubsection{Cluster 3 vs. cluster 1}

The disorders among the members of this cluster, who are mainly suffering from auditory and verbal disability, are influenced by a number of various causes. Cousin marriages, diseases during pregnancy, a family history of disability, childhood disease, head trauma, and traumas caused by war are directly related to the disability in this cluster $(\mathrm{P}<0.05)$. Nevertheless, head trauma and traumas caused by war, as the main causes increase the possibility of these disorders up to 29 to 39.3 times.

\subsubsection{Cluster 4 vs. cluster 1}

The participants in this cluster, with a wide range of disorders, are mostly influenced by traumas caused by car accidents, particularly head trauma, in a way that these causes increase the likelihood of these disability in this cluster up to 12.3 to 68.7 times. Moreover, the vulnerable members of this cluster are women $(\mathrm{P}<0.05)$.

\subsubsection{Cluster 5 vs. cluster 1}

The participants in this cluster are mainly suffering from visualauditory disability and spinal cord injuries. Trauma caused by car accidents is the only component related to the disability of this group and it increases the likelihood of disability up to 10 times.

The information presented in Table 4 shows the state of derived clusters with regards to a number of socio-demographic variables. The results in this table indicate that with regards to the distribution of age, education level, occupation, marital status, and residential location, there is a significant difference between the clusters $(P<0.001)$. 


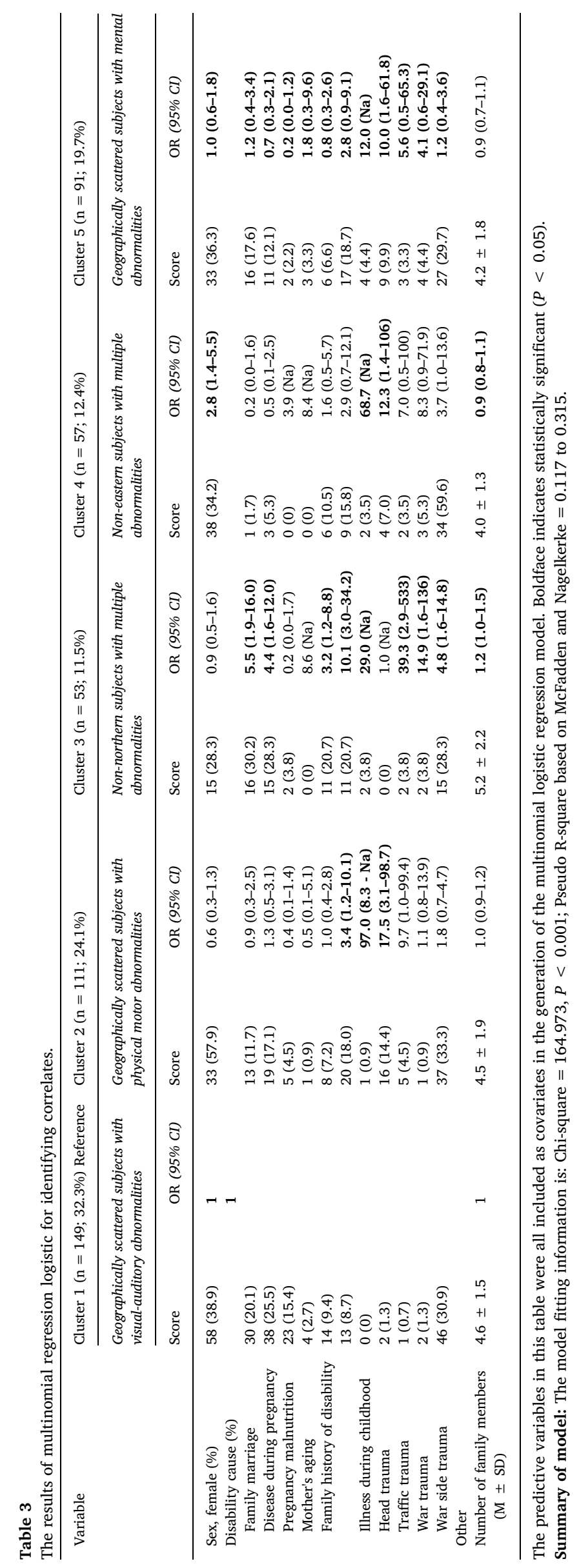


Table 4

The status of participants separated by the clusters.

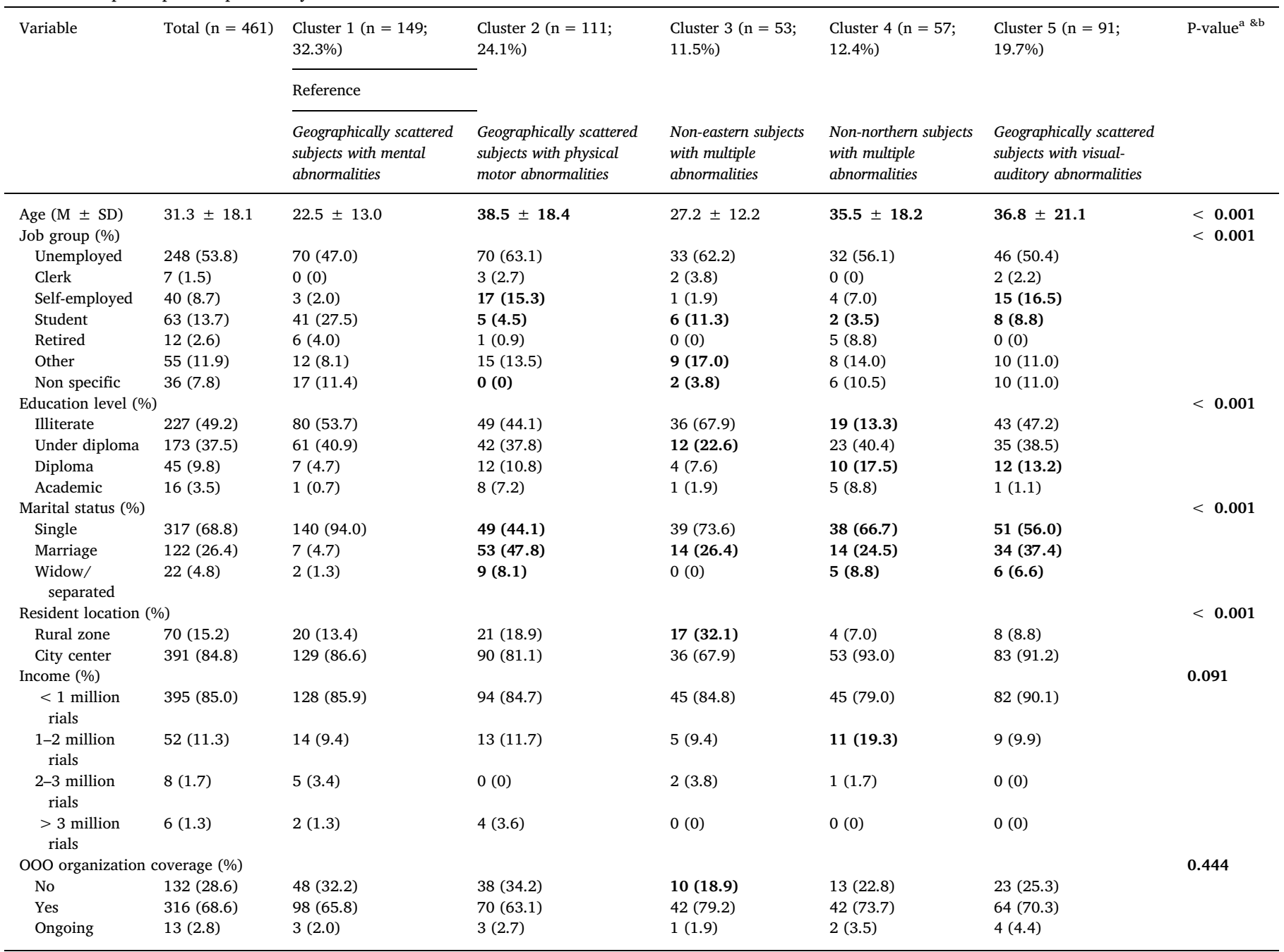

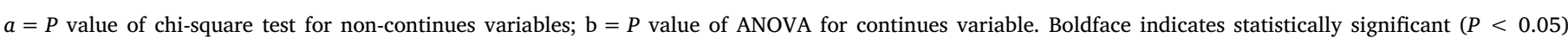
between each group with the reference group.

While generally there is no significant difference between the clusters with regards to income and financial support by the OOO Organization $(\mathrm{P}<0.05)$, compared to the reference cluster, a higher number of members of cluster 3 are financially supported by the OOO Organization.

\section{Discussion}

The current study was carried out in order to cluster various types of disability in Kermanshah Province and determine their predictive causes. The results show that with regards to the geographical dispersion of disability and their types, five clusters can be extracted from the model: (i) geographically scattered subjects with mental abnormalities, (ii) geographically scattered subjects with physical motor abnormalities, (iii) non-eastern subjects with multiple abnormalities, (iv) nonnorthern subjects with multiple abnormalities, and (v) geographically scattered subjects with visual-auditory abnormalities.

The results of the study show that compared to the reference cluster (people suffering from mental and psychological disorders), the members of cluster 2 are more vulnerable to childhood diseases, head trauma, and traumas caused by car accidents. In fact, childhood diseases and head trauma increase the likelihood of physical-motor disorders up to 3.4 to 17.5 times and head trauma increases this likelihood up to 97 times. Obviously, traumatic brain injuries are one of the most important causes of morbidity and disability caused by trauma. ${ }^{16}$ The frequency of these injuries is about 200 people per 100,000 people in developed countries and more than 500 people in 100,000 people in the U.S. ${ }^{17-19}$ It is estimated that 5.3 million people in USA are living with disability caused by head trauma. ${ }^{20}$ On the other hand, the results of a study shows that in low-income and average-income countries, $90 \%$ of physical disabilities and disability are caused by car accidents. ${ }^{21}$ According to the World Health Organization, car and road accidents are the main cause of morbidity and disability for people aged between three and 35 years. $^{22}$ Indeed, it can be claimed that traffic and car accidents are one of the main causes of disability and hospitalization, creating a huge economic and social load. ${ }^{23}$

The results of our study show that the disability among the members of the cluster 3, who are mainly suffering from auditory and verbal disability, is influenced by a number of various causes. Cousin marriages, diseases during pregnancy, a family history of disability, childhood diseases, head trauma, and traumas caused by war are all directly related to the disability in this cluster. Nevertheless, head trauma and traumas caused by war are the main causes which increase the likelihood of these disabilities up to 29 to 39.3 times. In order to justify these results, we can consider the geographical and economic position of the eastern cities of this province. Indeed, we can mention issues such 
as the eight-year war between Iran and Iraq and the undesirable economic and social conditions of these regions; however, the exact reasons for this result require more in-depth analysis. With regards to this issue, we can provide this hypothesis that it is possible that proximity to Iraq and issues such as chemical and microbial warfare in this country used by the two sides of the conflict in this country, and previously by Saddam's Regime, have led to the birth of children suffering from mental disorders. On the other hand, the undesirable economic and social conditions in these areas are one of the important factors that should be considered. Many scholars argue that the role of socioeconomic factors in disability is significant, ${ }^{24-27}$ since these factors can be related to malnutrition, socioeconomic inequalities, health problems, pre-pregnancy care, and health and education services, ${ }^{24-27}$ causing various types of disability.

Moreover, the results of the current study show that the participants in cluster 4 with a wide range of disability are mainly influenced by traumas caused by car accidents, particularly head trauma in a way that these causes increase the likelihood of these disorders in this cluster up to 12.3 to 68.7 times. Furthermore, the vulnerable members of this cluster are women. The interesting result in this section is the lack of a specific classification for the types of disability, which should be evaluated in depth. The majority of participants in this cluster are people living in the capital of the province, which include a wide range of people from different ethnic and racial backgrounds. Because of the increasing immigration into the capital of the province, the racial, linguistic, and cultural-religious population in this cluster is highly diversified. Due to the higher population of this area, and in turn heavier traffic, the number of car accidents in this area is very high. While evaluating the causes of disability in this cluster, in many cases, the option of "other causes" has a higher frequency than other options. Other causes are those who cannot be put into any of the categories. These items include causes such as diseases, strokes, burning and fires, sport accidents, falling from heights, occupational accidents, physical confrontations, and unknown causes. This may have been caused by the higher age and number of the elderly, the increase in diseases such as diabetes and MS, or issues such as the effects of mobile network electromagnetic waves, and the like and these issues must be considered while studying the causes of disability. However, on the other hand, a number of scholars believe that in $50 \%$ of the cases, the causes of disability are not known, ${ }^{28}$ and with regards to cluster 4 , we are facing this issue.

The results of the current study show that participants in the cluster 5 are mainly affected by visual-auditory disability and spinal cord injuries. Traumas caused by car accidents are the only factor related to the disability on this group, increasing the likelihood of disability up to 10 times. Car accidents are among factors that can be the main reason behind disability caused by spinal cord injuries. However, there can be a wide range of causes for visual and auditory disability. For instance, the results of a study on the relationship between cousin marriages and children's health in African and Asian countries show that cousin marriage is related to disorders such as deafness due to its effects through latent genes. ${ }^{29}$ Moreover, the results of another study show that $89 \%$ of the cases, blindness is caused by genetic factors and only $11 \%$ of the blindness cases are caused by external factors. ${ }^{30}$

Similar to other studies, the current study suffers from a number of limitations that may restrict the generalizability of its results. For instance, the sample selected for the current study included households in Kermanshah Province, so while generalizing the results to other societies; we must err on the side of caution. Moreover, considering the large size of the population, the sampling method was not random.

\section{Conclusion}

Based on the results of the current study, it can be said that the eastern areas of Kermanshah Province are mainly suffering from mental and psychological disorders. In western areas of this province, physical- motor disabilities are more frequent, and the central areas are characterized by visual-auditory disability. Moreover, the results of the current study show that mother's disease during pregnancy and cousin marriages are the most important factors causing mental-psychological disability. However, there are also a lot of unknown causes. Based on the findings of the current study and similar studies on the causes of disability, the design of comprehensive plans and programs for preventing disability seems necessary since it is proved that preventable factors affect the likelihood of disability. Scholars emphasize that prevention is very important and effective in reducing disability. This is why in many countries; the main focus is on preventive measures for various types of mental, physical, and sensory-motor disability. Since cousin marriages are among the important potential causes of disability, and considering the cultural and social fabric of Kermanshah Province, the issues with this type of marriage must be taught and discussed among the population of this province.

\section{Conflicts of interest}

None of the authors have conflicts of interest to report.

\section{Funding}

Welfare Organization of Kermanshah Province (ID: 1396).

\section{Ethical approval}

Not required.

\section{Acknowledgment}

We would like to express our deepest gratitude to the authorities of the Welfare Organization of Kermanshah Province for their financial support of the current study. We appreciate the Clinical Research Development Center of Imam Reza Hospital, Kermanshah University of Medical Sciences.

\section{References}

1. Lucas-Carrasco R, Eser E, Hao Y, McPherson KM, Green A, Kullmann L. The quality of care and support (QOCS) for people with disability scale: development and psychometric properties. Res Dev Disabil. 2011;32(3):1212-1225.

2. Gladman JR. The international classification of functioning, disability and health and its value to rehabilitation and geriatric medicine. J Chin Med Assoc. 2008;71(6):275-278

3. Barbotte E, Guillemin F, Chau N, Lordisability G. Prevalence of impairments, disabilities, disability and quality of life in the general population: a review of recent literature. Bull World Health Organ. 2001;79(11):1047-1055.

4. Nasiripour AA, Tabibi J, Afkar A, Kamali M. Effect of community based rehabilitation program implementation on disabled people living conditions in Iran. $J$ Knowl Health. 2012;7(4):173-178http://knh.shmu.ac.ir/index.php/site/article/view/75.

5. Chala MB, Mekonnen S, Andargie G, et al. Prevalence of disability and associated factors in dabat health and demographic surveillance system site, northwest Ethiopia. BMC Publ Health. 2017;17(1):762.

6. Mitra S, Sambamoorthi U. Disability prevalence among adults: estimates for 54 countries and progress toward a global estimate. Disabil Rehabil. 2014;36(11):940-947.

7. Hansen N, Philo C. The normality of doing things differently: bodies, spaces and disability geography. $J$ Econ Soc Geogr. 2007;98(4):493-506https://onlinelibrary. wiley.com/doi/pdf/10.1111/j.1467-9663.2007.00417.x.

8. Velayutham B, Kangusamy B, Joshua V, Mehendale S. The prevalence of disability in elderly in India - analysis of 2011 census data. Disabil Health J. 2016;9(4):584-592.

9. Wong E, Woodward M, Stevenson C, Backholer K, Sarink D, Peeters A. Prevalence of disability in Australian elderly: impact of trends in obesity and diabetes. Prev Med. 2016;82:105-110,

10. Khandaker G, Muhit M, Rashid H, et al. Infectious causes of childhood disability: results from a pilot study in rural Bangladesh. J Trop Pediatr. 2014;60(5):363-369.

11. King G, Petrenchik T, DeWit D, McDougall J, Hurley P, Law M. Out-of-school time activity participation profiles of children with physical disabilities: a cluster analysis. Child Care Health Dev. 2010;36(5):726-741.

12. Trevithick L, Painter J, Keown P. Mental health clustering and diagnosis in psychiatric in-patients. BJPsych Bull. 2015;39(3):119-123.

13. Bucher CO, Dubuc N, von Gunten A, Trottier L, Morin D. Development and validation of clinical profiles of patients hospitalized due to behavioral and psychological 
symptoms of dementia. BMC Psychiatr. 2016;16(1):261.

14. van den Berge MJC, Free RH, Arnold R, et al. Cluster analysis to identify possible subgroups in tinnitus patients. Front Neurol. 2017;8(115):1-7.

15. Larsson B, Gerdle B, Bernfort L, Levin LA, Dragioti E. Distinctive subgroups derived by cluster analysis based on pain and psychological symptoms in Swedish older adults with chronic pain - a population study (PainS65 +). BMC Geriatr. 2017;17(1):200.

16. Saatman KE, Duhaime A-C, Bullock R, Maas AI, Valadka A, Manley GT. Classification of traumatic brain injury for targeted therapies. J Neurotrauma. 2008;25(7):719-738.

17. Bruns J, Hauser WA. The epidemiology of traumatic brain injury: a review. Epilepsia. 2003;44(s10):2-10.

18. Thurman DJ. The epidemiology of traumatic brain injury in children and youths: a review of research since 1990. $J$ Child Neurol. 2016;31(1):20-27. https://doi.org/10. $1177 / 0883073814544363$.

19. Bazarian JJ, Mcclung J, Shah MN, Ting Cheng Y, Flesher W, Kraus J. Mild traumatic brain injury in the United States, 1998-2000. Brain Inj. 2005;19(2):85-91.

20. Langlois JA, Rutland-Brown W, Wald MM. The epidemiology and impact of traumatic brain injury: a brief overview. J Head Trauma Rehabil. 2006;21(5):375-378.

21. Jacobs JB, Sayer I. Road accident in developing countries. Accid Anal Prev. 2003;15(5):337-353.

22. Duperrex O, Bunn F, Roberts I. Safety education of pedestrians for injury prevention: a systematic review of randomised controlled trials. BMJ. 2002;324(7346):1129.

23. Bahadorimonfared A, Soori $\mathrm{H}$, Mehrabi $\mathrm{Y}$, et al. Trends of fatal road traffic injuries in Iran (2004-2011). PloS One. 2013;8(5):e65198.

24. Kidd MP, Sloane PJ, Ferko L. Disability and the labour market: an analysis of British males. J Health Econ. 2000;19:961-981.

25. Mitra S, Posara A, Vick B. Disability and poverty in developing countries: a multidimensional study. World Dev. 2013;41:1-18.

26. Robertson JM, Hatton C, Emerson E. The identification of children with or at significant risk of intellectual disabilities in low and middle income countries: a review. J Appl Res Intellect Disabil. 2012;25:99-118.

27. Maulik PK, Darmstadt GL. Childhood disabilities in low and middle income countries: overview of screening, prevention, services, legislation and epidemiology. Pediatrics. 2007;120(Suppl. 1):S1-S55.

28. Santos C, Rodriguez-Revenga L, Madrigal I, Badenas C, Pineda M, Milà M. A novel mutation in JARID1C gene associated with mental retardation. Eur J Hum Genet. 2006;14(5):583

29. Saggar AK, Bittles AH. Consanguinity and child health. Paediatr Child Health. 2008;18(5):244-249.

30. Amgad AK, Ehab FH, Khalid FT. Childhood blindness at a school for the blind in Riyadh, Saudi Arabia. Ophthalmic Epidemiol. 2006;13(1):1-5. 